\title{
Semi-automatic Brain Tumor Segmentation by Constrained MRFs Using Structural Trajectories*
}

\author{
Liang Zhao ${ }^{1}$, Wei $\mathrm{Wu}^{2}$, and Jason J. Corso ${ }^{1}$ \\ 1 Computer Science and Engineering, SUNY at Buffalo, Buffalo, NY, USA \\ 2 Wuhan University of Science and Technology, Wuhan, Hubei, China \\ $\{$ lzhao6, jcorso\}@buffalo.edu
}

\begin{abstract}
Quantifying volume and growth of a brain tumor is a primary prognostic measure and hence has received much attention in the medical imaging community. Most methods have sought a fully automatic segmentation, but the variability in shape and appearance of brain tumor has limited their success and further adoption in the clinic. In reaction, we present a semi-automatic brain tumor segmentation framework for multi-channel magnetic resonance (MR) images. This framework does not require prior model construction and only requires manual labels on one automatically selected slice. All other slices are labeled by an iterative multi-label Markov random field optimization with hard constraints. Structural trajectories - the medical image analog to optical flow-and 3D image over-segmentation are used to capture pixel correspondences between consecutive slices for pixel labeling. We show robustness and effectiveness through an evaluation on the 2012 MICCAI BRATS Challenge Dataset; our results indicate superior performance to baselines and demonstrate the utility of the constrained MRF formulation.
\end{abstract}

\section{Introduction}

Magnetic resonance imaging provides detailed information of the human brain and is an essential tool for the neuro-oncologist. Quantifying the volume of a brain tumor is the key prognostic measurement of tumor progression [1, 2]. Yet, manually labeling a brain tumor in 3D MRI is a time-consuming and error-prone task. The medical imaging community has hence invested a significant amount of effort in methods for automatic brain tumor segmentation [3-7]. However, despite these efforts, there has been limited success in translation to the clinical environment; the current performance of automatic methods does not meet the prognostic needs [1] (e.g., the best performer in BRATS 2012 has a Jaccard score of 0.5 for high-grade tumor). This may be due to the underlying variability of tumor shape and appearance, or due to assumptions of the approaches.

Semi-automatic methods that require some input from the user are a plausible alternative. They have received comparatively little attention for brain tumor

\footnotetext{
* This work was partially supported by the Chinese National Science Foundation (61273241) and the NSF CAREER grant IIS-0845282.
} 
imaging, e.g., [2, 8, 9]. In contrast, 2D image interactive labeling has been hotly studied, e.g., GrabCut [10], LazySnapping [11]. Similar ideas have been used on 2D brain tumor segmentation, such as [8]. However, generalizing these ideas to $3 \mathrm{D}$ is non-trivial. The interaction mechanisms in these papers, such as drawing lines to specify foreground and background, moving a square brush or lasso, or giving a bounding box, are no longer trivial tasks in a $3 \mathrm{D}$ volume for complex objects, tumor and edema. Recently, some approaches for object segmentation in 3D medical images by iterative energy optimization based on shape model were proposed [12, 13]. However, compared to some organs, such as the liver, the edema and tumor shape are comparatively harder to model.

We propose an alternative mechanism for incorporating human input in semiautomatic brain tumor segmentation. Our main idea is based on the assumption that pixels with the same label in consecutive slices will have a similar feature distribution and strong spatial correspondence. Hence, we require the human to manually label only one slice (using standard 2D annotation techniques) and then all other slices are sequentially labeled based on a constrained Markov random field model. The constraints in the model are created based on a 3D notion of optical flow, which we call structural trajectories, and on over-segmentation. The initial slice to be labeled is automatically selected based on an asymmetry heuristic. Our thorough experiments on the BRATS 2012 data set [3] demonstrate the potential of our approach with limited input of only one manually labeled slice (our Jaccard score for high-grade tumor is 0.75 , which is a $50 \%$ improvement over the best fully automatic method). To the best of our knowledge, this is the first time an optical flow-like calculation has been used to provide a global 3D structural consistency measure for brain tumor segmentation.

\section{Methods}

Our proposed semi-automatic segmentation approach labels the volume sliceby-slice using a constrained Markov random field (MRF) energy minimization on neighboring slices. In this procedure, the structural correspondence between adjacent slices is detected by optical flow estimation, which we call structural trajectories and helps in the iterative pixel labeling as hard constraints.

Given a sequence of slices, $S=\left\{s_{i}, i=1: n\right\}$, the whole procedure of the proposed approach is as follows:

1. Compute structural trajectories $T R=\left\{t r_{j}, j=1 \ldots M\right\}$.

2. Select the most asymmetric slice $s_{i_{\max }}$.

3. Label pixels in $s_{i_{\max }}$ as tumor, edema, or background manually.

4. In $s_{i_{\max }+1}$ to $s_{n}$ and $s_{i_{\max }-1}$ to $s_{1}$, label pixels slice-by-slice with Alg. 1 .

\subsection{Slice Selection by Asymmetric Area Detection}

Consider a 3D MRI brain image, $I m g$. We first flip it sagittally, yielding $I m g_{\text {mirror }}$ and then refine it with a non-rigid 3D registration [14] to the original $I m g$. We then compare the refined flipped image $I m g_{r}$ to $I m g$ to look for regions of high 


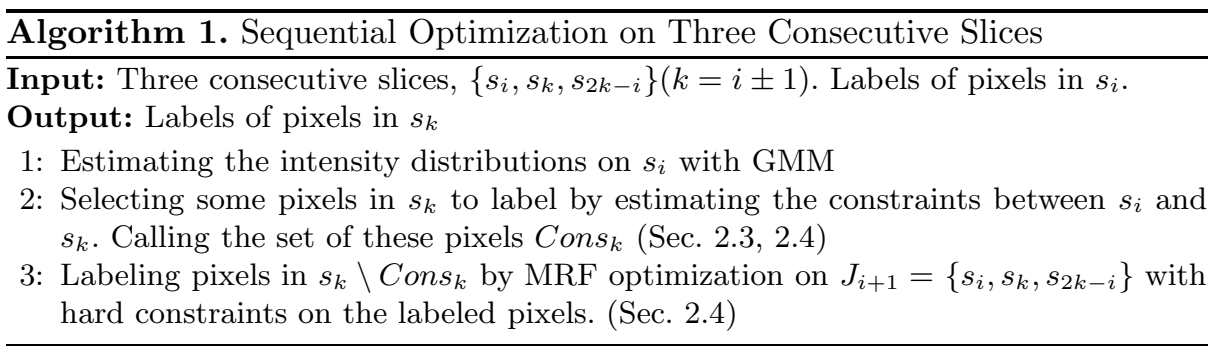

asymmetry. Concretely, for pixel $p \in \operatorname{Img}(x, y, z)$, let $I(p)$ be its feature vector (4D MRI). To define $\operatorname{Asym}(p)$, we measure local asymmetry of $p$ :

$$
\operatorname{Asym}(p)=\min _{q \in N(p)}\left\|I(p)-\operatorname{Img}_{r}(q)\right\|
$$

where, $N(p)$ is the neighborhood of $p$. We use Otsu's Method [15] on $\operatorname{Asym}(p)$, $p \in I$, resulting in the threshold $\theta_{\text {Aysm }}$ and define the most asymmetric slice: $i_{\text {max }}=\arg \min _{i} \sum_{p \in s_{i}} \mathbf{1}\left(\operatorname{Asym}(p) \geq \theta_{\text {Aysm }}\right)$, where $\mathbf{1}(\cdot)$ returns 1 if the argument is true and 0 otherwise.

\subsection{Annotation}

For manual annotation of the selected slide, we have developed an interface that allows the human to make labels (Tumor, Edema and Background) in all four channels. The interface supports drawing rectangles and curves with the mouse. When a pixel is labeled in one channel, it will be labeled in all the other three channels in the same time in the interface. This approach has been a minimal burden on the user as the asymetric slice typically finds large tumor and edema regions and at the same time, our method is robust to minor errors in initial labeling (see Sec. 2.4 for details). A novice annotator with no background on medicine spends about 2-4 minutes (used in this paper).

\subsection{Structural Trajectories}

To exploit the 3D structural consistency of the MRI volume, we develop a method to constrain the ultimate pixel label problem based on tracking voxels through the volume, which we call structural trajectories. Ultimately, these will form constraints for our MRF formulation (i.e., linking a tumor pixel in one slice to the corresponding tumor pixel in the next slice, if it exists, and analogously for the other types of tissue). We use an optical flow algorithm [16] to track points between neighboring slices by successive registrations, resulting in spatially dense trajectories, capturing the global correspondence of pix-

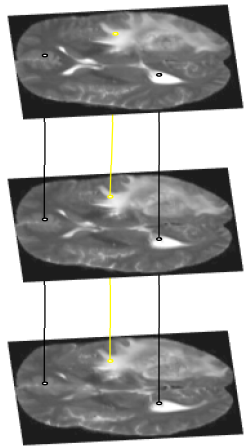

Fig. 1. Example trajectories els over the whole volume.

First, we register neighboring slices by minimizing intensity and gradient pixel matching scores [17]. Given a sequence of slices $S=\left\{s_{i}, i=1 \ldots n\right\}$, define a structural trajectory to be a sequence of pixels: $t r_{j}=\left\{p_{t}^{j} \mid p_{t}^{j} \in s_{t}, t=t_{0} \ldots t_{1}, 1 \leq t_{0} \leq\right.$ 


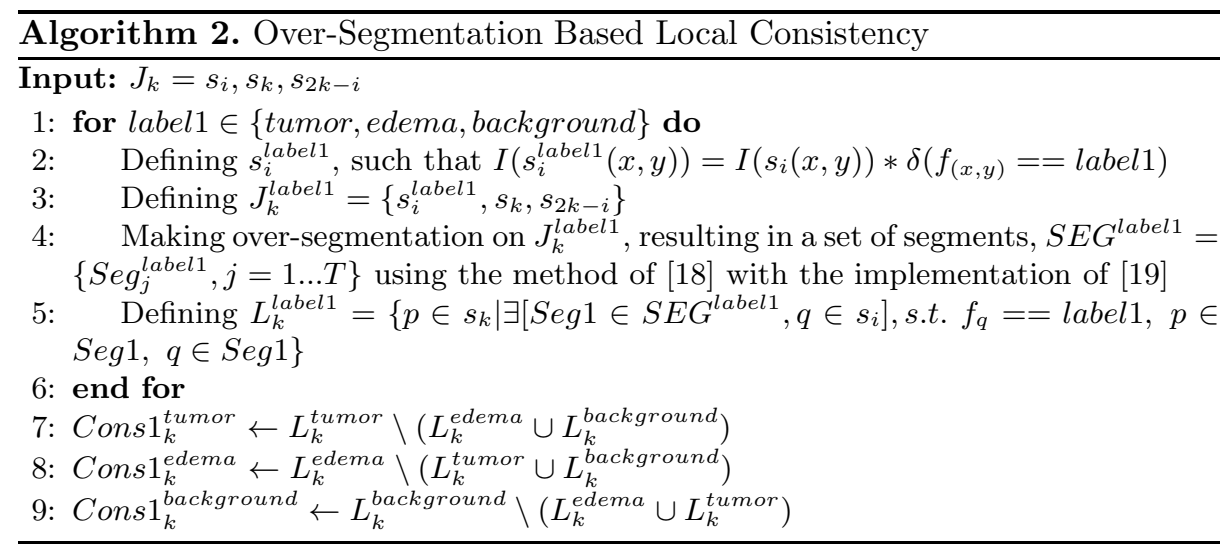

$\left.t_{1} \leq n\right\}$. Trajectories hence capture slice-to-slice pairwise pixel correspondence (Fig. 1), even if the pair of pixels are not neighboring. In a brain MRI 3D image, a point trajectory refers to a sequence of physical points in brain which intensities are successively similar. We use the point tracking with forward-backward checking method to compute the trajectories. To the best of our knowledge, this is the first time an optical flow-like computation has been used to enforce structural consistency in 3D MRI brain tumor segmentation.

\subsection{Constrained MRF Pixel Labeling}

Given the label of pixels of $s_{i}$, we define a traditional Markov random field on pixels of $J_{k}=\left\{s_{i}, s_{k}, s_{2 k-i}\right\}$. $(k=i \pm 1)$. In this pixel labeling problem we have a $3 \mathrm{D}$ image $J_{k}$ with a 6 -connected neighborhood system $N$ and a set of labels $L=\{$ tumor, edema,background $\}$. A labeling $f$ assigns a label $f_{p} \in L$. The feature vector of $p$ is defined as $I(p)$. The goal is to find a labeling minimizing an energy function of the form,

$$
E(f)=\sum_{p \in J_{k}} D_{p}\left(f_{p}\right)+\sum_{(p, q) \in N} V_{p q}\left(f_{p}, f_{q}\right)
$$

where $D_{p}\left(f_{p}\right)=-\log \left(P\left(I(p) \mid f_{p}\right)\right)$ is the data term and smoothness is

$$
V_{p q}\left(f_{p}, f_{q}\right)=\operatorname{Smoothness}\left(f_{p}, f_{q}\right) \times \exp (-\alpha * \operatorname{Dis}(I(p), I(q), \Sigma))
$$

where Smoothness is a $3 \times 3$ non-negative matrix, $\alpha$ is a positive scalar, Dis $(\cdot)$ is Mahalanobis distance, $\operatorname{Dis}(x, y, \Sigma)=\sqrt{(x-y)^{\top} \Sigma^{-1}(x-y)} . \Sigma$ is computed with the training data.

Estimation of Intensity Distribution: Using the labeled pixels in $s_{i}$ as training data, we model the node-class likelihoods $P\left(I(p) \mid f_{p}\right)$ as a Gaussian mixture (GMM). Let $\Sigma$ be the covariance matrix of $\left\{I(p) \mid p \in s_{i}, f_{p}==\right.$ background $\}$.

Estimation of Constraints: With the constraints between $s_{i}$ and $s_{k}$, we use two different methods to select two sets of pixels in $s_{j}$ to label; here a constraint 


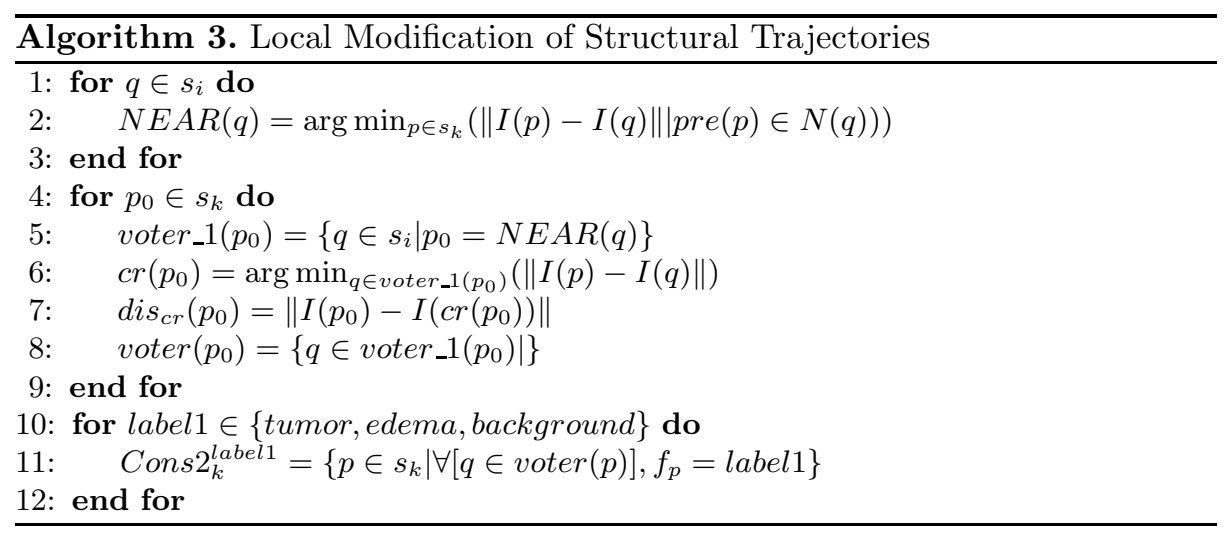

means that we will fix the label of the pixel during inference use it to propagate the labels to neighboring unconstrained pixels (see Sec. 2.5 for details).

Erosion-like Processing with Over-Segmentation: The basic idea is that if a pixel is grouped (in the over-segmentation) with other pixels of different labels on $s_{i}$, then its labeling has a high uncertainty and it should hence not be selected as a hard-constraint, which makes our method robust to minor errors in the labeling. The estimation process is performed according to Alg. 2.

Local Modification of Structural Trajectories: Structuraltrajectories cross slices and there is strong correspondence between the pixels in the same trajectory. We make a local modification of these trajectories to select some pixels in $s_{k}$ to label. For a pixel $p_{0} \in s_{k}$, let $\operatorname{pre}\left(p_{0}\right)$ be the pixel in $s_{i}$ and in the same trajectory with $p_{0}$. Obviously, $p_{0}$ is likely to have the same label with $\operatorname{pre}\left(p_{0}\right)$. Let $\operatorname{voter}\left(p_{0}\right)$ be the set of pixels in $s_{i}$ that have the same label with $p_{0}$. Define $\operatorname{Cons} 2_{k}^{\text {label } 1}$ as the set of pixels in $s_{k}$ that we select to label as label1. Alg. 3 shows how to compute Cons $2_{k}^{\text {label1 }}$ and Fig. 2 gives a visualization. We then intersect the two constraint sets, Cons $s_{k}^{\text {label } 1}=$ Cons $1_{k}^{\text {label } 1} \cap$ Cons $1_{k}^{\text {label } 1}$ where

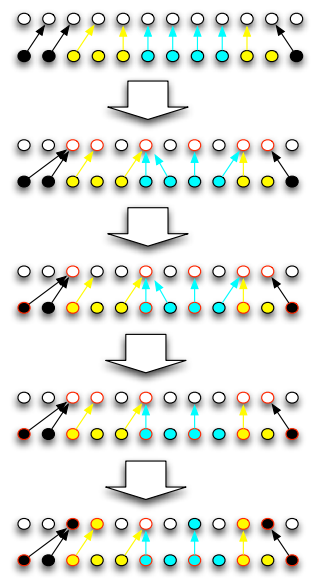

Fig. 2. Local modification of structural trajectories label $1 \in\{$ tumor, edema,background $\}$ and Cons $_{k}=\cup$ Cons $s_{k}^{\text {label } 1}$, as a conservative step since these will be used as hard constraints in the MRF optimization (all pixels in Cons ${ }_{k}^{\text {label1 }}$ are given label label1), which we discuss next.

\subsection{Optimizing MRF with Hard Constraints}

In $J_{k}=\left\{s_{i}, s_{k}, s_{2 k-i}\right\}$, we have pixels in $s_{i} \cup$ Cons $_{k}$ labeled. Hence, the Markov random field on $J_{k}$ is constrained by the labeled pixels. We implement these hard constraints through with the following MRF conversion. We define $H_{k}=$ 
$s_{i} \cup$ Cons $_{k}, J_{k}^{H}=J_{k} \backslash H_{k}$. We can define a new MRF on $J_{k}^{H}$ with the same neighborhood system $N$, which has no hard constraints. To make the two MRFs equivalent, we define a new energy function based on the original one in Eq. (2):

$$
\begin{aligned}
E^{H}\left(f^{H}\right) & =\sum_{p \in J_{k}^{H}} D_{p}^{H}\left(f_{p}^{H}\right)+\sum_{(p, q) \in N} V_{p q}^{H}\left(f_{p}^{H}, f_{q}^{H}\right) . \\
D_{p}^{H}\left(f_{p}^{H}\right) & =D_{p}\left(f_{p}^{H}\right)+\sum_{(p, q) \in N, q \in H} V_{p q}\left(f_{p}^{H}, f_{q}^{H}\right) \\
V_{p q}^{H}\left(f_{p}^{H}, f_{q}^{H}\right) & =V_{p q}\left(f_{p}^{H}, f_{q}^{H}\right)
\end{aligned}
$$

It is easy to prove, if $\forall p \in J_{k}^{H}, f_{p}=f_{p}^{H}$, then $E^{H}\left(f^{H}\right)=E(f)$. Hence, we can optimize $E(f)$ on $J_{k}$ by optimizing $E^{H}\left(f^{H}\right)$ on $J_{k}^{H}$. Our MRF is a conventional multi-class MRF, we hence optimize with the alpha-expansion graph cut [20].

\section{$3 \quad$ Experiments and Results}

Data Set: We evaluate our method on 2012 BRATS Training Data [3], which is now the standard benchmark for brain tumor segmentation research. This dataset contains 30 cases from patients with high- and low-grade gliomas. The data has four channels, T1, T2, T1C and FLAIR; the feature $I(p)$ in our experiment is hence a $4 \mathrm{D}$ vector. The typical volume size is about $130 \times 170 \times 170$. We also evaluate our method on 2012 BRATS Challenge Data [3], which contains 11 high- and 4 low-grade gliomas from patients, and compare the result with a state-of-the-art semi-automatic method, Tumor-cut [9]. The gold standard labels are not given and DICE scores of Tumor and Complete Tumor(Tumor+Edema) are computed by an online evaluation infrastructure (hence we avoid a bias by the human annotator, who has never seen the gold standard). The DICE Scores of [9] are given by 2012 BRATS [3].

Parameters: The MRF parameter $\alpha$ is set to 0.001 and Smoothness is given by Table 1 based on empirical experiments.

Baselines and Metrics: We use the Jaccard and DICE metrics in our evaluation. $j^{*}$

Table 1. Smoothness term

\begin{tabular}{|c|c|c|c|}
\hline Smoothness & bg & tumor & edema \\
\hline bg & 0 & 2 & 1 \\
\hline tumor & 2 & 0 & 1 \\
\hline edema & 1 & 1 & 0 \\
\hline
\end{tabular}
refers to our method. We define a set of appropriate baselines: to evaluate the hard constraints from the structural trajectories and the over-segmentation, denote the baseline without any hard constraints as $j^{B}$. We also assess the value of the structural trajectories, which are global through the whole volume, with respect to the local over-segmentation constraints; $j^{L}$ uses only the local over-segmentation. We follow the exact evaluation regime specified in [3].

Results: We show the quantitative results in Table 2, As a point of comparison to the current state of the art fully automatic method, we also compare our results to supervised automatic method [21] on the same dataset (denoting it $j^{A}$ ), which won the first prize in the BRATS Challenge 2012 [3]. Although this 
Table 2. Comparative Jaccard scores on BRATS Training Set and DICE scores on BRATS Challenge Set: our method $\left(j^{*}\right)$, no hard constraints $\left(j^{B}\right)$, no structural trajectories $\left(j^{L}\right)$, the best fully automatic method [21] from [3] $\left(j^{A}\right)$, and the state-of-the-art semi-automatic Tumor-cut $\left(j^{T}\right)$ []

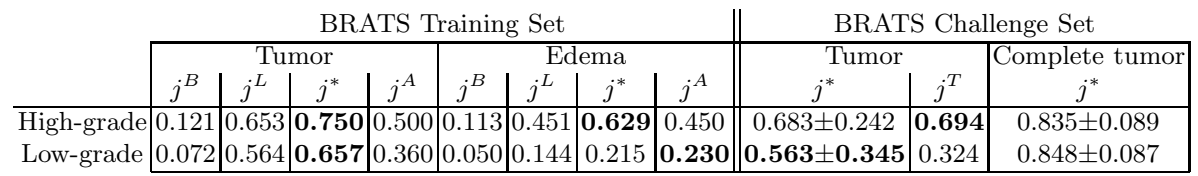

comparison will clearly favor our semi-automatic method, we show it to get a sense of what value our approach yields. Note that not all of the semi-automatic methods in Table 2 outscore the automatic counterparts. The results clearly demonstrate that the proposed semi-automatic method, which requires only one slice to be labeled by a human, outperforms the best fully automatic method 21] by a significant margin on all cases except the low-grade edema class, which is known to be challenging. The results also indicate the value of both the local over-segmentation constraints and the global structural trajectories. The average runtime of one case is about 5 minutes in Matlab. Fig. 3 shows an example.

Failure Modes: Mode 1: In some cases, especially low-grade cases, tumor or edema is not successive sliceby-slice. In these cases, because we cannot estimate the feature distribution accurately and no pixel correspondence is available, our results may just contain some connected components of tumor or edema. Mode 2: Let $A$ be a small homogeneous region contained in slice $s_{k}$. If its feature distribution is very different from any of the feature distribution of tu-

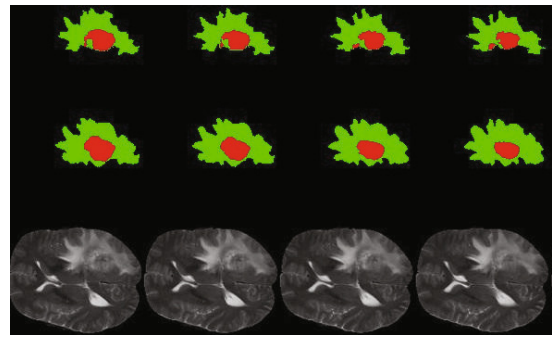

Fig. 3. Example results. Top: results of our method (Tumor: Red; Edema: Green). Middle: gold standard. Bottom: T1C-MRI. mor, edema or background estimated by the previous labeled slice $s_{i}$, and it is strongly adjacent to both of two different tissues (e.g. tumor and edema), then the pixel labeling of $A$ by MRF optimization will not be accurate.

\section{Conclusion}

In this work, we have proposed a novel semi-automatic brain tumor segmentation method. We just need to ask for a manual labeling on a single slice, which is selected automatically based on asymmetry. All the other slices are labeled iteratively with the local intensity distribution and both global and local constraints in a constrained MRF framework. The constraints are extracted automatically 
based on an optical flow-like measure, which is the first time such a structural global measure has been used for brain tumor segmentation, to the best of our knowledge. Our method does not depend on the assumption that training data and testing data have similar intensity distribution or similar shape prior, unlike many existing methods. Our experiments demonstrate that the proposed approach yields significant improvements over fully automatic methods (e.g., 50\%) in most cases, as expected, and the novel structural constraints greatly improve the MRF optimization over the conventional MRF labeling.

\section{References}

1. Patel, M.R., Tse, V.: Diagnosis and staging of brain tumors. Seminars in Roentgenology 39(3), 347-360 (2004)

2. Liu, J., Udupa, J.K., Odhner, D., Hackney, D., Moonis, G.: A system for brain tumor volume estimation via mr imaging and fuzzy connectedness. CMIG 29(1), 21-34 (2005)

3. Menze, B., Jakab, A., Bauer, S., Reyes, M., Leemput, K.V.: Proceedings of the miccai 2012 challenge on multimodal brain tumor segmentation (2012)

4. Corso, J.J., Sharon, E., Dube, S., El-Saden, S., Sinha, U., Yuille, A.: Efficient multilevel brain tumor segmentation with integrated bayesian model classification. IEEE Trans. on Medical Imaging 27(5), 629-640 (2008)

5. Prastawa, M., Bullitt, E., Ho, S., Gerig, G.: A brain tumor segmentation framework based on outlier detection. MEDIA 8, 275-283 (2004)

6. Lee, C.-H., Schmidt, M., Murtha, A., Bistritz, A., Sander, J., Greiner, R.: Segmenting brain tumors with conditional random fields and support vector machines. In: Liu, Y., Jiang, T.-Z., Zhang, C. (eds.) CVBIA 2005. LNCS, vol. 3765, pp. 469-478. Springer, Heidelberg (2005)

7. Geremia, E., Zikic, D., Clatz, O., et al.: Classification forests for semantic segmentation of brain lesions in multi-channel mri. Decision Forests for Computer Vision and Medical Image Analysis (2013)

8. Birkbeck, N., Cobzas, D., Jägersand, M., Murtha, A., Kesztyues, T.: An interactive graph cut method for brain tumor segmentation. In: WACV (2009)

9. Hamamci, A., Kucuk, N., Karaman, K., Engin, K., Ünal, G.B.: Tumor-cut: Segmentation of brain tumors on contrast enhanced $\mathrm{mr}$ images for radiosurgery applications. IEEE Trans. Med. Imaging 31(3), 790-804 (2012)

10. Rother, C., Kolmogorov, V., Blake, A.: Grabcut -interactive foreground extraction using iterated graph cuts. In: SIGGRAPH (2004)

11. Li, Y., Sun, J., Tang, C.K., Shum, H.Y.: Lazy snapping. In: SIGGRAPH (2004)

12. Chen, X., Yao, J., Zhuge, Y., Bagci, U.: 2d automatic anatomy segmentation based on graph cut-oriented active appearance models. In: ICIP (2010)

13. Afifi, A., Nakaguchi, T.: Liver segmentation approach using graph cuts and iteratively estimated shape and intensity constrains. In: Ayache, N., Delingette, H., Golland, P., Mori, K. (eds.) MICCAI 2012, Part II. LNCS, vol. 7511, pp. 395-403. Springer, Heidelberg (2012)

14. Ourselin, S., Stefanescu, R., Pennec, X.: Robust registration of multi-modal images: Towards real-time clinical applications. In: Dohi, T., Kikinis, R. (eds.) MICCAI 2002, Part II. LNCS, vol. 2489, pp. 140-147. Springer, Heidelberg (2002)

15. Otsu, N.: A threshold selection method from gray-level histograms. IEEE Transactions on Systems, Man and Cybernetics (1979) 
16. Sundaram, N., Brox, T., Keutzer, K.: Dense point trajectories by GPU-accelerated large displacement optical flow. In: Daniilidis, K., Maragos, P., Paragios, N. (eds.) ECCV 2010, Part I. LNCS, vol. 6311, pp. 438-451. Springer, Heidelberg (2010)

17. Brox, T., Bruhn, A., Papenberg, N., Weickert, J.: High accuracy optical flow estimation based on a theory for warping. In: Pajdla, T., Matas, J(G.) (eds.) ECCV 2004, Part IV. LNCS, vol. 3024, pp. 25-36. Springer, Heidelberg (2004)

18. Grundmann, M., Kwatra, V., Han, M., Essa, I.A.: Efficient hierarchical graphbased video segmentation. In: CVPR (2010)

19. Xu, C., Corso, J.J.: Evaluation of super-voxel methods for early video processing. In: CVPR (2012)

20. Boykov, Y., Veksler, O., Zabih, R.: Fast approximate energy minimization via graph cuts. IEEE Trans. Pattern Anal. Mach. Intell. 23(11), 1222-1239 (2001)

21. Bauer, S., Fejes, T., Slotboom, J., Wiest, R., Nolte, L.P., Reyes1, M.: Segmentation of brain tumor images based on integrated hierarchical classification and regularization. In: MICCAI BRATS Challenge (2012) 